\section{Preferência alimentar sanguínea de flebotomíneos da Amazônia do Maranhão, Brasil}

\author{
Feeding preference of sand flies in the Amazon, \\ Maranhão State, Brazil
}

\author{
1 Departamento de Biologia, \\ Universidade Federal do \\ Maranhão, São Luís, Brasil. \\ 2 Departamento de Patologia, \\ Universidade Federal do \\ Maranhão, São Luís, Brasil. \\ ${ }^{3}$ Instituto Oswaldo Cruz, \\ Fundação Oswaldo Cruz, \\ Rio de Janeiro, Brasil. \\ Correspondência \\ J. M. M. Rebêlo \\ Laboratório de Entomologia \\ e Vetores, Departamento \\ de Patologia, Universidade \\ Federal do Maranhão. \\ Praça Madre Deus 2, São \\ Luís, MA 65025-560, Brasil. \\ macariorebelo@uol.com.br
}

\section{Abstract}

The objective of this study was to determine the blood feeding sources for sand flies based on the precipitin test in Buriticupu, Amazon Region, Maranhão State, Brazil, from July 2002 to June 2004. Single (87.6\%) and double (8\%) reactions and non-reactive samples (4.4\%) were found. The presence of sand flies fed on human blood (6.7\%) and blood of animals that are possible peridomiciliary Leishmania reservoirs helps explain the reporting of autochthonous tegumentary leishmaniasis cases in Buriticupu.

Lutzomyia; Phlebotomine; Leishmaniasis
Yrla Nívea Oliveira-Pereira 1

Jorge Luiz Pinto Moraes 2

Elias Seixas Lorosa 3

José Manuel Macário Rebêlo 1,2

\section{Introdução}

Nos flebotomíneos, a hematofagia é um hábito exclusivo das fêmeas, que necessitam do sangue para a maturação dos ovários. Ao realizar a hematofagia as fêmeas podem transmitir protozoários do gênero Leishmania entre mamíferos, em determinados ambientes. O caráter oportunista dos flebotomíneos no que diz respeito à alimentação sanguínea, revela uma grande variedade de vertebrados que podem funcionar como fonte alimentar 1,2 .

O estudo do conteúdo estomacal de flebotomíneos é de grande significado ecológico e epidemiológico. Primeiro, porque permite descobrir a identidade dos hospedeiros sanguíneos, segundo, porque serve como subsídio para a indicação de reservatórios potenciais de leishmânias e o papel protetor que certos animais poderiam desempenhar, em relação ao homem, em área de transmissão destes parasitas. Nesse sentido, pode nortear as atividades de controle e vigilância sanitária.

Neste trabalho investigou-se a fonte alimentar sanguínea de flebotomíneos e a presença de animais domésticos e sinantrópicos no peridomicílio, em área de transmissão de leishmaniose tegumentar. 


\section{Métodos}

O estudo foi realizado em Buriticupu (4 ${ }^{\circ} 27^{\prime} 22^{\prime \prime}$ $4^{\circ} 30^{\prime} 00^{\prime \prime}$ Sul e $46^{\circ} 35^{\prime} 27^{\prime \prime}-46^{\circ} 54^{\prime} 03^{\prime \prime}$ Oeste), o município da Amazônia do Maranhão, Brasil, que apresentou o maior coeficiente médio de detecção de casos de leishmaniose tegumentar $(862,6)$, no período de 1997 a 2001, segundo dados do Ministério da Saúde 3 .

Foram selecionadas duas casas do povoado rural da Sexta Vicinal, nas proximidades de uma capoeira. No paiol de cada casa foi instalada uma armadilha CDC, sem a presença de animais domésticos como iscas. Os flebotomíneos foram coletados das 18 às 6 horas, uma vez por mês, no período de julho de 2002 a junho de 2004. Assim, cada armadilha funcionou 288 horas. Considerando-se as duas armadilhas juntas, o esforço de captura foi de 576 horas.

As espécies de flebotomíneos foram identificadas por caracteres morfológicos 4 e o teste da reação da precipitina foi aplicado ao conteúdo intestinal das fêmeas previamente alimentadas com sangue de vertebrados. O tubo digestório dos espécimes foi retirado e o conteúdo estomacal foi triturado em salina a $0,85 \%$. Esse macerado foi deixado por 12 horas à temperatura entre 4o e 8०C e logo após centrifugado por 5 minutos a 1.500rpm; o sobrenadante foi exposto a anti-so- ros de humano, ave, boi, cão, cavalo, cabra, gato, porco, roedor, mucura (Didelphis), tatu, carneiro, lagarto e sapo.

A presença no peridomicílio de animais sinantrópicos e domésticos, e a distância de abrigos destes últimos em relação às habitações humanas foram investigadas por meio da aplicação de questionário, nas 45 habitações que constituíam o povoado.

\section{Resultados}

No total foram encontradas 274 fêmeas ingurgitadas, distribuídas entre Lutzomyia choti (164 exemplares), L. triacantha (90) e L. whitmani (20), conforme Tabela 1. Dessas, $240(87,6 \%)$ apresentaram reações simples: roedor $(40 \%)$, ave $(25,8 \%)$, eqüino $(11,7 \%)$, cão $(6,7 \%)$, humano (6,7\%), boi (5\%) e mucura (4,2\%); e em 22 (8\%) foram detectadas reações duplas: ave/mucura (27,3\%), ave/cão (18,2\%), ave/humano (18,2\%), roedor/ave $(18,2 \%)$, boi/cão $(9,1 \%)$ e humano/ roedor $(9,1 \%)$.

As fêmeas de L. choti haviam ingerido sangue de todos os hospedeiros, mas principalmente de roedor (40\%), ave (24\%), cão (9,3\%) e eqüino (9,3\%); as de L. triacantha só não sugaram o sangue de mucura, preferindo o de roedor $(38,9 \%)$,

Tabela 1

Reações simples e duplas das fontes alimentares sanguíneas de flebotomíneos da Sexta Vicinal. Município de Buriticupu, Amazônia Maranhense, Brasil, 2002-2004.

\begin{tabular}{|c|c|c|c|c|c|c|c|c|}
\hline \multirow[t]{2}{*}{ Hospedeiros } & \multicolumn{2}{|c|}{ Lutzomyia choti } & \multicolumn{2}{|c|}{ Lutzomyia triacantha } & \multicolumn{2}{|c|}{ Lutzomyia whitmani } & \multicolumn{2}{|c|}{ Total } \\
\hline & $n$ & $\%$ & $\mathrm{n}$ & $\%$ & $\mathrm{n}$ & $\%$ & $n$ & $\%$ \\
\hline Ave & 36 & 24,0 & 24 & 33,3 & 2 & 11,1 & 62 & 25,8 \\
\hline Boi & 6 & 4,0 & 4 & 5,5 & 2 & 11,1 & 12 & 5,0 \\
\hline Cão & 14 & 9,3 & 2 & 2,8 & - & - & 16 & 6,7 \\
\hline Eqüino & 14 & 9,3 & 10 & 13,9 & 4 & 22,2 & 28 & 11,7 \\
\hline Humano & 10 & 6,7 & 4 & 5,5 & 2 & 11,1 & 16 & 6,7 \\
\hline Mucura & 10 & 6,7 & - & - & - & - & 10 & 4,2 \\
\hline Roedor & 60 & 40,0 & 28 & 38,9 & 8 & 44,4 & 96 & 40,0 \\
\hline Subtotal & 150 & 62,5 & 72 & 30,0 & 18 & 7,5 & 240 & 87,6 \\
\hline Ave/Cão & 4 & 33,3 & - & - & - & - & 4 & 18,2 \\
\hline Ave/Humano & - & - & 2 & 25,0 & 2 & 100,0 & 4 & 18,2 \\
\hline Ave/Mucura & 2 & 16,7 & 4 & 50,0 & - & - & 6 & 27,3 \\
\hline Boi/Cão & - & - & 2 & 25,0 & - & - & 2 & 9,1 \\
\hline Humano/Roedor & 2 & 16,7 & - & - & - & - & 2 & 9,1 \\
\hline Roedor/Ave & 4 & 33,3 & - & - & - & - & 4 & 18,2 \\
\hline Subtotal & 12 & 54,5 & 8 & 36,4 & 2 & 9,1 & 22 & 8,0 \\
\hline Não reagiram & 2 & 16,7 & 10 & 83,3 & - & - & 12 & 4,4 \\
\hline Total & 164 & 59,8 & 90 & 32,8 & 20 & 7,3 & 274 & 100,0 \\
\hline
\end{tabular}


ave (33,3\%) e eqüino (13,9\%); enquanto as de $L$. whitmani utilizaram menos hospedeiros, preferindo roedor $(44,4 \%)$ e eqüino $(22,2 \%)$, e sugaram mais sangue humano (11,1\%) do que $L$. choti (6,7\%) e L. triacantha (5,5\%). Nas reações duplas, as aves foram mais procuradas $(66,7 \%)$ e $L$. choti apresentou mais reações (4) do que $L$. triacantha (3) e L. whitmani (1).

Os animais domésticos mais citados pela população entrevistada foram: galinha (87\% das residências), cão $(55,6 \%)$, porco $(31,1 \%)$, gato $(26,7 \%)$, jumento $(11,1 \%)$, pato $(8,9 \%)$, papagaio $(4,4 \%)$ e boi $(2,2 \%)$. Esses animais somaram 687 indivíduos e a galinha representou $74,1 \%$, seguida pelo porco (12,2\%), cão (8\%), gato (2,9\%), pato e jumento ( $1 \%)$, papagaio e boi $(0,7 \%)$. As distâncias dos abrigos de animais para as residências variaram de 5 a 50 metros, mas a maioria (36,3\%) encontrava-se a cerca de 20 metros.

Os mamíferos sinantrópicos encontrados no peridomicílio foram: morcego (58,5\%), mucura $(12,2 \%)$, raposa $(12,2 \%)$, mucuri (Caluromys) $(7,3 \%)$, roedor $(5 \%)$, gato do mato e tatu $(4,8 \%)$, além de lagarto $(84,4 \%)$, cobra $(77,8 \%)$ e sapo (60\%).

\section{Discussão}

As fêmeas de flebotomíneos examinadas neste estudo preferiram sangue de roedor, mas sugaram ave, com certa freqüência, além de outros vertebrados; um dado interessante que reforça a necessidade de investigação de alguns destes animais como possíveis reservatórios de leishmaniose tegumentar, especialmente os mais procurados, como roedores, cães e eqüinos, os quais já foram encontrados infectados com Leishmania em outras áreas 5,6,7.
A julgar pela maior freqüência no peridomicílio, à semelhança das observações obtidas no Município de Raposa 1, também no Maranhão, as galinhas adquirem importância como hospedeiros sanguíneos, pois não existem evidências de que possam funcionar como reservatório de leishmânias.

As distâncias dos abrigos de animais domésticos para as residências estavam dentro do raio de dispersão dos flebotomíneos 8, um aspecto relevante, pois quanto mais próximos estiverem delas, mais fácil será o acesso do vetor ao intradomicílio, considerando o desenvolvimento da antropofilia pelos flebotomíneos e o tipo das habitações encontradas, caracteristicamente feitas de taipa ou de madeira.

O encontro no peridomicílio de exemplares de Lutzomyia alimentados, ao mesmo tempo, com sangue humano, de animais sinantrópicos (roedor e mucura) e de animais domésticos (cão e eqüino), corrobora a hipótese de que a transmissão da infecção leishmaniótica esteja ocorrendo neste ambiente.

Observou-se neste estudo que $L$. whitmani sugou menos hospedeiros que as outras duas espécies, mas este achado deve estar ligado ao número menor de exemplares capturados e não reduz a sua importância epidemiológica, pois os estudos de infecção por Leishmania 9 em flebotomíneos obtidos no ambiente peridoméstico das mesmas casas estudadas neste trabalho detectaram positividade apenas para as fêmeas de L. whitmani, espécie reconhecidamente antropofílica ${ }^{10}$. Nesse caso, é possível que todos os elos da cadeia de transmissão de Leishmania estejam presentes no peridomicílio do povoado de Sexta Vicinal, o que, em última análise, explica a existência de casos autóctones de leishmaniose tegumentar. 


\section{Resumo}

O objetivo deste estudo foi determinar as fontes alimentares sanguineas de flebotomíneos por meio da reação da precipitina, em Buriticupu, na Amazônia maranhense, Brasil, de julho de 2002 a junho de 2004. Foram detectadas reações simples (87,6\%), duplas (8\%) $e$ não reagentes $(4,4 \%)$. A presença de flebotomíneos alimentados com sangue humano (6,7\%) e de possíveis reservatórios de Leishmania no peridomicílio ajuda a explicar a ocorrência de casos autóctones de leishmaniose tegumentar no Município de Buriticupu.

Lutzomyia; Phlebotominae; Leishmaniose

\section{Colaboradores}

Y. N. Oliveira-Pereira e J. L. P. Moraes participaram do planejamento das atividades, coleta e identificação dos flebotomíneos, organização dos dados e revisão do manuscrito. E. S. Lorosa fez os exames da reação da precipitina, interpretação dos dados e revisão do manuscrito. Coube a J. M. M. Rebêlo a coleta dos flebotomíneos, análise dos dados e elaboração do artigo.

\section{Agradecimentos}

Trabalho financiado pelo Conselho Nacional de Desenvolvimento Científico e Tecnológico (CNPq).

\section{Referências}

1. Dias FOP, Lorosa ES, Rebêlo JMM. Fonte alimentar sangüínea e a peridomiciliação de Lutzomyia longipalpis (Lutz \& Neiva, 1912) (Psychodidae, Phlebotominae). Cad Saúde Pública 2003; 19:1373-80.

2. Nery LCR, Lorosa ES, Franco AMR. Feeding preference of the sand flies Lutzomyia umbratilis and $L$. spathotrichia (Diptera: Psychodidae. Phlebotominae) in an urban forest patch in the city of Manaus, Amazonas, Brazil. Mem Inst Oswaldo Cruz 2004; 99:571-4.

3. Ministério da Saúde. Vigilância e monitoramento da leishmaniose tegumentar americana em unidades territoriais - Brasil, 1994 - 2001. Boletim Eletrônico Epidemiológico 2002; 2(5).

4. Young DG, Duncan MA. Guide to the identification and geographic distribution of Lutzomyia sandflies in Mexico, the West Indies; Central and South America (Diptera: Psychodidae). Memoirs of the American Entomology Institute 1994; 54:1-881.

5. Aguilar CM, Rangel EF. Leishmaniose tegumentar em uma mula (Equus caballus $\mathrm{x}$ Equus asinus) em área endêmica no Estado do Rio de Janeiro. Mem Inst Oswaldo Cruz 1986; 81:239-40.

6. Falqueto A, Coura JR, Barros GC, Grimaldi Filho G, Sessa PA, Carias VRD, et al. Participação do cão no ciclo de transmissão da leishmaniose tegumentar no Município de Viana, Estado do Espírito Santo, Brasil. Mem Inst Oswaldo Cruz 1986; 81:155-63.
7. Silveira FT, Lainson R, Shaw JJ, Braga R, Ishikawa EAY, Souza AAA. Leishmaniose cutânea na Amazônia: isolamento de Leishmania (Viannia) lainsoni do roedor Agouti paca (Rodentia: Dasyproctidae), no Estado do Pará, Brasil. Rev Inst Med Trop São Paulo 1991; 33:18-22.

8. Morrison AC, Ferro C, Morales A, Tesh RB, Wilson ML. Dispersal of the sand fly Lutzomyia longipalpis (Diptera: Psychodidae) at an endemic focus of visceral leishmaniasis in Colombia. J Med Entomol 1993; 30:427-35.

9. Oliveira-Pereira YNO, Rebêlo JMM, Moraes JLP, Pereira SRFP. Diagnóstico molecular da taxa de infecção natural de flebotomíneos (Psychodidae, Lutzomyia) por Leishmania sp. na Amazônia maranhense. Rev Soc Bras Med Trop 2006; 39:540-3.

10. Campbell-Lendrum DH, Pinto MC, Brandão-Filho SP, Souza AA, Ready PD, Davies CR. Experimental comparison of anthropophily between geographically dispersed populations of Lutzomyia whitmani (Diptera: Psychodidae). Med Vet Entomol 1999; 13:299-309.

Recebido em 05/Mar/2008

Versão final reapresentada em 18/Jun/2008 Aprovado em 26/Jun/2008 